\title{
SPECTRAL TYPES OF UNIFORM DISTRIBUTION
}

\author{
GEON H. CHOE
}

(Communicated by J. Marshall Ash)

\begin{abstract}
We investigate the spectral types of unitary operator $U$ on $L^{2}(\mathbb{T})$ defined by $(U f)(x)=A(x) f(x+\theta),|A(x)|=1$ a.e., where $\mathbb{T}$ is the unit circle identified with the half open interval $[0,1)$ and $\theta$ is irrational. It is shown that Veech's result on the Kronecker-Weyl theorem modulo 2 is closely related to the spectral type of $U$.
\end{abstract}

\section{INTRODUCTION}

Let $\mathbb{T}$ be the unit circle $|z|=1$ identified with the half open interval $[0,1)$. Take an irrational number $\theta \in \mathbb{T}$ and consider the rotation by $\theta$. The classical Kronecker-Weyl theorem states that, for a given interval $I \subset \mathbb{T}$,

$$
\lim _{n \rightarrow \infty} \frac{1}{n} \sum_{k=0}^{n-1} \chi_{I}(k \theta)=m(I)
$$

where $m$ is the normalized Lebesgue measure on the circle. In 1969, Veech [13] initiated the study of the following problem: Let $y_{n} \equiv \sum_{k=0}^{n-1} \chi_{I}(k \theta)(\bmod$ 2), $y_{n} \in\{0,1\}$, and consider the existence and value of the limit

$$
\lim _{N \rightarrow \infty} \frac{1}{N} \sum_{1}^{N} y_{n}
$$

One might expect that the limit exists and equals $\frac{1}{2}$. But contrary to our intuition, he proved that the limit always exists if and only if $\theta$ has bounded partial quotients in its continued fraction expansion. (For the theory of continued fractions, see [9].) The limit might not be equal to $\frac{1}{2}$ if $\exp \left(\pi i \chi_{I}(x)\right)$ is a coboundary, that is, $\exp \left(\pi i \chi_{I}(x)\right)=\overline{q(x)} q(x+\theta)$ for some $q(x)$ of modulus 1 a.e. on the circle. Veech showed that for $\theta$ with bounded partial quotients $\exp \left(\pi i \chi_{I}(x)\right)$ is a constant multiple of a coboundary if and only if $t \in \mathbb{Z} \cdot \theta$ where $t$ is the length of the interval $I$. But for $\theta$ with unbounded partial quotients the same is true for uncountably many $t$ 's.

Received by the editors December 2, 1991 and, in revised form, July 7, 1992.

1991 Mathematics Subject Classification. Primary 11K06, 28D05, 47A35.

Key words and phrases. Maximal spectral type, uniform distribution modulo 1, coboundary.

Research partially supported by GARC-KOSEF. 
In this paper we investigate the same problem from a spectral theoretic viewpoint rather than seeing what happens at a single point when the limit (2) does not exist or is not equal to $\frac{1}{2}$. We formulate the problem in the following general setting: Let $(X, \mu)$ be a probability space and $\tau$ be a $\mu$-invariant ergodic transformation on $X$, which is not necessarily invertible. We consider the behavior of the sequence $y_{n}(x) \equiv \sum_{k=0}^{n-1} \chi_{I}\left(\tau^{k} x\right)(\bmod 2), y_{n}(x) \in\{0,1\}$, at each point $x$ of $X$ where $I$ is a measurable subset in $X$. Equivalently, we consider the sequence $2 y_{n}(x)-1, n=1,2,3, \ldots$, at each $x$ and check whether the limit approaches zero. Note that $2 y_{n}(x)-1=\exp \left(\pi i y_{n}(x)\right)$. Now we define an isometry $U$ on $L^{2}(X)$ by

$$
(U f)(x)=\exp \left(\pi i \chi_{I}(x)\right) f(\tau x)
$$

Then, for $n \neq 1$,

$$
\left(U^{n} f\right)(x)=\exp \left(\pi i \sum_{k=0}^{n-1} \chi_{I}\left(\tau^{k} x\right)\right) f\left(\tau^{n} x\right)
$$

and

$$
\left(U^{n} 1\right)(x)=\exp \left(\pi i \sum_{k=0}^{n-1} \chi_{I}\left(\tau^{k} x\right)\right)=\exp \left(\pi i y_{n}(x)\right),
$$

and now our problem is to study the existence of

$$
\lim _{N \rightarrow \infty} \frac{1}{N} \sum_{n=1}^{N}\left(U^{n} 1\right)(x) \text {. }
$$

By von Neumann's Mean Ergodic Theorem, we have that $\frac{1}{N} \sum_{n=1}^{N}\left(U^{n} 1\right)(x)$ converges to $P_{H} 1$ in $L^{2}(X)$ where $P_{H}$ is the orthogonal projection of the constant function 1 onto the invariant subspace $H=\left\{h \in L^{2}(X): U h=h\right\}$.

If an isometry $U f(x)=A(x) f(\tau x),|A(x)|=1$ a.e., has an eigenvalue $\lambda$, then we can choose $q \in L^{2}(X)$ such that $\|q\|_{2}=1, U q=\lambda q$. Hence $A(x) q(\tau x)=\lambda q(x)$. Since $|A(x)|=1$, we have $|q(x)|=|\lambda||q(\tau x)|$, and $|q|$ is an eigenfunction of $U$ and $|\lambda|$ is an tigenvalue. Since $\tau$ is ergodic, we see that $|q|$ is constant a.e. and $|\lambda|=1$. Hence $A(x)=\lambda \overline{q(\tau x)} q(x)$. Recall that a function $f(x)$ is called a coboundary if $f(x)=\overline{q(\tau x)} q(x)$ where $|q(x)|=1$ a.e. on $X$. Therefore $U$ has an eigenvalue if and only if $A(x)$ is a constant multiple of a coboundary. And also note that $f$ is a coboundary if and only if $\bar{f}$ is a coboundary.

For an invertible ergodic transformation $\tau$ we have the induced unitary operator $U_{\tau}$ defined by $U_{\tau} f(x)=f(\tau x)$. In this case, $(U f)(x)=A(x) f(\tau x)$, $|A(x)|=1$ a.e., is also unitary.

The operator $U$ also appears in ergodic theory: Let $G$ be a compact abelian group with Haar measure $\nu$ and $\phi: X \rightarrow G$ be a measurable function. We define the skew product transformation $\tau_{\phi}$ on the product probability space $(X \times G, \mu \times \nu)$ by $\tau_{\phi}(x, g)=(\tau x, g \cdot \phi(x))$. Note that $\tau_{\phi}$ is also measurepreserving. The transformation $\tau_{\phi}: X \times G \rightarrow X \times G$ is not ergodic if and only if there is a nontrivial character $\chi$ of $G$ and a measurable map $\eta$ of $X$ into the unit circle $\mathbb{T}$ satisfying $\chi(f(x))=\eta(x) \overline{\eta(\tau x)}$. In the case that $G=\mathbb{T}$ and $\phi: X \rightarrow \mathbb{T}$, we have the induced operator

$$
(V h)(x, y)=h\left(\tau_{\phi}(x, y)\right)=h(\tau x, y+\phi(x))
$$


for $h \in L^{2}(X \times \mathbb{T}), x \in X, y \in \mathbb{T}$. It is easy to see that $L^{2}(X \times \mathbb{T})$ is the orthogonal sum of the $V$-invariant subspaces $H_{n}=\left\{h(x) e^{2 \pi i n y}: h \in L^{2}(X)\right\}$, $n=0,1,2, \ldots$. Note that $\left.V\right|_{H_{0}}$ is unitarily equivalent to $U_{\tau}$ and that $\left.V\right|_{H_{1}}$ is unitarily equivalent to $U$.

We are mainly concerned with the spectral types of unitary operators. The following definitions are needed. Let $U=\int_{|z|=1} z d P(z)$ be the spectral representation of a unitary operator $U$ in a separable Hilbert space $H$ where $P$ is the corresponding spectral measure on the unit circle. Recall that $P(E)$ is an orthogonal projection in $H$ for every measurable set in the unit circle. If $C(f)=\operatorname{span}\left\{U^{n} f: n \in \mathbb{Z}\right\}$ is the cyclic subspace generated by $f \in H$, then the spectral theorem says that there exists a sequence $f_{1}, f_{2}, f_{3}, \ldots$ in $H$ such that $\sigma_{f_{1}} \gg \sigma_{f_{2}} \gg \sigma_{f_{3}} \gg \cdots$ and $H=C\left(f_{1}\right) \oplus C\left(f_{2}\right) \oplus C\left(f_{3}\right) \oplus \cdots$ where $\sigma_{f}$ is a positive finite measure on the unit circle defined by $E \mapsto(P(E) f, f)$. The sequence $f_{1}, f_{2}, f_{3}, \ldots$ is unique in the sense that for any other sequence $g_{1}, g_{2}, g_{3}, \ldots$ in $H$ satisfying $\sigma_{g_{1}} \gg \sigma_{g_{2}} \gg \sigma_{g_{3}} \gg \cdots$ and $H=C\left(g_{1}\right) \oplus C\left(g_{2}\right) \oplus C\left(g_{3}\right) \oplus \cdots$ we have $\sigma_{f_{i}} \approx \sigma_{g_{i}}$ for every $i$. The spectral type of $\sigma_{f_{1}}$ is called the maximal spectral type of $U$. We say that $U$ has continuous spectrum if $\sigma_{f_{1}}$ is a continuous measure and that $U$ has discrete spectrum if $\sigma_{f_{1}}$ is a discrete measure. Note that a unitary operator $U$ has discrete part in its spectrum if and only if it has an eigenvalue, which is necessarily of modulus 1 . If $\sigma_{f_{1}}$ is equivalent to the Lebesgue measure, then $U$ is said to have Lebesgue spectrum. And if $\sigma_{f_{1}}$ and the Lebesgue measure are mutually singular, then $U$ is said to have singular spectrum.

\section{SPECTRUM AND UNIFORM DISTRIBUTION}

The following result shows when $U$ and $U_{\tau}$ have the same spectral type.

Proposition 1. For an invertible ergodic transformation $\tau$ we let $U_{\tau}$ be the unitary operator defined by $U_{\tau} f(x)=f(\tau x)$. Then the spectrum of the unitary operator $(U f)(x)=A(x) f(\tau x),|A(x)| \equiv 1$ a.e., has nontrivial discrete part if and only if $A(x)$ is a constant multiple of a coboundary. And in this case, $U$ and $U_{\tau}$ are have the same spectral types.

Proof. Let $U=\int_{|\lambda|=1} \lambda d P(\lambda)$ be the spectral representation of the unitary operator $U$ where $P$ is the corresponding spectral measure. Suppose that there exists $0 \neq f \in L^{2}(X)$ such that the measure $E \mapsto(P(E) f, f)$ defined on $|\lambda|=1$ has discrete part. Then for some $\lambda_{0}$ we have $\left(P\left(\left\{\lambda_{0}\right\}\right) f, f\right) \neq 0$. In other words, $P\left(\left\{\lambda_{0}\right\}\right) \neq 0$. Hence there exists $q,\|q\|_{2}=1$ satisfying $P\left(\left\{\lambda_{0}\right\}\right) q=q$. Note that $P\left(\left\{\lambda_{0}\right\}^{c}\right) q=0$. Therefore, $U q=\int_{|\lambda|=1} \lambda d P(\lambda) q=\lambda_{0} P\left(\left\{\lambda_{0}\right\}\right) q=\lambda_{0} q$ and $A(x) q(\tau x)=\lambda_{0} q(x)$. Since $|A(x)| \equiv 1$, we have $|q(x)|=|q(\tau x)|$ and now ergodicity of $\tau$ implies $|q| \equiv 1$. Hence $A(x)=\lambda_{0} \overline{q(\tau x)} q(x)$.

Now we suppose that $A(x)=\lambda \overline{q(x)} q(\tau x)$ for some $q,|q(x)|=1$ a.e. and $\lambda,|\lambda|=1$. Since $U \overline{q(x)}=A(x) \overline{q(\tau x)}=\lambda \overline{q(x)} q(\tau x) \overline{q(\tau x)}=\lambda \overline{q(x)}, U$ has an eigenvector $\bar{q}$, and its spectrum has nontrivial discrete part.

To show the spectral equivalence, we let $V$ be the unitary operator defined by the multiplication by the function $q$ of modulus 1 . Then $V U=\lambda U_{\tau} V$ and $U$ and $\lambda U_{\tau}$ are spectrally equivalent. Hence $U$ and $U_{\tau}$ have the same spectral types. 
Proposition 2. Let $\tau$ be an ergodic transformation on $X$, and let $U$ be the isometry on $L^{2}(X)$ given by $U f(x)=A(x) f(\tau x)$ where $A(x)$ is real-valued and $|A(x)|=1$ a.e. on $X$. Define a subspace $H=\left\{h \in L^{2}(X): U h=h\right\}$. Then the dimension of $H$ is 0 or 1 . Furthermore, the following hold:

(i) If $\operatorname{dim} H=0$, then

$$
\frac{1}{N} \sum_{n=1}^{N} U^{n} 1 \rightarrow 0 \quad \text { in } L^{2}(X) .
$$

(ii) If $\operatorname{dim} H=1$, then $A(x)$ is a coboundary. And the converse is also true. In this case, if we take $q \in H,\|q\|_{2}=1$, then $|q(x)| \equiv 1$ a.e. and $A(x)=\overline{q(x)} q(\tau x)$. Furthermore, we may choose a real-valued function for such $q$. In this case,

$$
\frac{1}{N} \sum_{n=1}^{N} U^{n} 1 \rightarrow \int_{X} q(x) d \mu \cdot q \quad \text { in } L^{2}(X) .
$$

Proof. To find the dimension of $H$, we take $f \neq 0, g \neq 0 \in H$. Then $U f=f, U g=g$. Since $A(x)=1, f(\tau x) \overline{g(\tau x)}=A(x) f(\tau x) A(x) \overline{g(\tau x)}=$ $(U f)(x)(U \bar{g})(x)=f(x) \overline{g(x)}$. Hence $f \bar{g}$ is constant because of the ergodicity of $\tau$. Now we apply von Neumann's Mean Ergodic Theorem in $L^{2}(\mathbb{T})$. From $U q=q$, we have $A(x) q(\tau x)=q(x)$, and by taking the absolute values we see that $|q(x)|$ is constant. Since $\|q\|_{2}=1$, we have $|q(x)| \equiv 1$ and $A(x)=$ $\overline{q(x)} q(\tau x)$ by taking the complex conjugates of both sides. Now by taking the squares of $A(x)=\overline{q(x)} q(\tau x)$, we obtain $1=\overline{q^{2}(x)} q^{2}(\tau x), q^{2}(x)=q^{2}(x+\tau)$. Now the ergodicity of $\tau$ implies that $q^{2}(x)$ is a constant of modulus 1 and we put $q 2(x)=\bar{\lambda}^{2},|\lambda|=1$. Then $(\lambda q)^{2} \equiv 1, \lambda q(x)=1$. Putting $p(x)=\lambda q(x)$, we see that $A(x)=\overline{p(x)} p(\tau x)$.

Remark. When the limit (2) fails to converge to zero, (ii) provides us with information on the extent of irregularity. That is, $\frac{1}{N} \sum_{1}^{N}\left(U^{n} 1\right)(x)$ converges to a real-valued function of modulus $\left|\int_{X} q(x) d \mu\right|$. And the size of $\int_{X} q(x) d \mu$ can be estimated as follows:

Proposition 3. Let $\tau$ be an ergodic transformation on a probability space $(X, \mu)$. Suppose $A(x)=\exp \left(\pi i \chi_{I}(x)\right)$ where $I$ is a measurable set in $X$. If $\operatorname{dim} H=1$ and $q \in H$ satisfies $A(x)=q(x) q(\tau x), q(x)$ real, then $\left|\int_{X} q(x) d\right| \leq 1-(I)$. Proof. Since $q(x)$ is real, it is of the form $q(x)=\exp \left(\pi i \chi_{E}(x)\right)$ for some measurable set $E$ in $X$. Then $\exp \left(\pi i \chi_{I}(x)\right)=\exp \left(\pi i \chi_{E}(x)\right) \exp \left(\pi i \chi_{\tau^{-1} E}(x)\right)$ and $I=E \Delta \tau^{-1} E$ modulo measure zero sets where $\Delta$ denotes the symmetric difference of two sets. Hence

$$
\mu(I)=\mu\left(E \Delta \tau^{-1} E\right) \leq \mu(E)+\mu\left(\tau^{-1} E\right)=2 \mu(E)
$$

and

$$
\int_{X} \mu(x) d \mu=1 \cdot(1-\mu(E))+(-1) \cdot \mu(E)=1-2 \mu(E) \leq 1-\mu(I) .
$$

On the other hand, we have

$$
\exp \left(\pi i \chi_{I}(x)\right)=(-q)(x)(-q)(\tau x)=\exp \left(\pi i \chi_{F}(x)\right) \exp \left(\pi i \chi_{\tau^{-1} F}(x)\right)
$$


where $F$ is the complement of $E$ in $\mathbb{T}$. In this case, $I=F \Delta \tau^{-1} F$ modulo measure zero sets and

$$
\mu(I)=\mu\left(F \Delta \tau^{-1} F\right) \leq \mu(F)+\mu\left(\tau^{-1} F\right)=2 \mu(F)=2(1-\mu(E))=2-2 \mu(E) .
$$

Hence

$$
\int_{X} q(x) d x=1-2 \mu(E) \neq \mu(I)-1 .
$$

Therefore $\left|\int_{X} q(x) d \mu\right| \leq 1-\mu(I)$.

Remark. (i) Roughly speaking, the larger the size of the set $I$, the less the extent of the irregularity of convergence to 0 , which fits our intuition.

(ii) Note that $\exp \left(\pi i \chi_{I}(x)\right)$ is a coboundary if and only if there exists a measurable set $E \in X$ such that $I=E \triangle \tau^{-1} E$ modulo measure zero sets. For, if there exists a real-valued function $q$ such that $\exp \left(\chi_{I}(x)\right)=q(x) q(\tau x)$, then we let $q=\exp \left(\pi i \chi_{E}\right)$ and this implies the required condition.

\section{KRONECKER-WEYL THEOREM}

To study Veech's problem on the Kronecker-Weyl theorem modulo 2, we consider the transformation $\tau_{\theta}: x \mapsto x+\theta, \theta$ irrational, and the corresponding unitary operator $U f(x)=\exp \left(\pi i \chi_{I}(x)\right)$ where $I$ is an interval. Proposition 2(i) is nothing new since it follows from Veech's original result on the pointwise convergence to zero. But when the limit (2) does not converge to zero, then (ii) provides us with a certain amount of information on the extent of irregularity. That is, $\frac{1}{N} \sum_{1}^{N}\left(U^{n} 1\right)(x)$ converges to $a$ where $|a| \leq 1-$ length $(I)$. Note that Veech's original result on the irregularity of the convergence, which contradicts our intuition, now does not seem to be so contradictory if we consider the fact that the larger the size of the set $I$, the less the extent of the irregularity of convergence, which fits our intuition more or less.

It is easy to see that $\exp \left(\pi i \chi_{[0,1 / 2 k)}\right)$ is not a constant multiple of a coboundary, where $k$ is a fixed positive integer. For, if it were of the form

$$
\exp \left(\pi i \chi_{[0,1 / 2 k)}\right)=\lambda \overline{q(x)} q(x+\theta),
$$

then by squaring both sides we would have $1=\lambda^{2} \overline{q^{2}(x)} q^{2}(x+\theta)$; hence, $\bar{\lambda}^{2} q^{2}(x)=q^{2}(x+\theta)$ and $\bar{\lambda}^{2}$ would be an eigenvalue of the irrational rotation by $\theta$. Hence $\bar{\lambda}^{2}=e^{2 \pi i n \theta}$ for some $n \in \mathbb{Z}$. Now if we take the product of the translates

$\exp \left(\pi i \chi_{[i-1 / 2 k, i / 2 k)}\right)=\lambda \overline{q(x-(i-1) / 2 k)} q(x-(i-1) / 2 k+\theta), \quad 1 \leq i \leq 2 k$, then we obtain

$$
-1=\exp \left(\pi i \chi_{[0,1)}\right)=\lambda^{2 k} \overline{Q(x)} Q(x+\theta)
$$

for some $Q(x)$; hence, $-\bar{\lambda}^{2 k} Q(x)=Q(x+\theta)$ and $-\bar{\lambda}^{2 k}=-e^{2 \pi i n k \theta}$ is an eigenvalue of the rotation by $\theta$, which is a contradiction. In fact, Conze [4] showed that $\exp \left(\pi i \chi_{[0, r)}\right)$ is not a constant multiple of a coboundary when $0<r<1$ and $r$ is a rational number.

For examples of coboundaries, consider the case when the length of the interval $I$ is given by integral multiples of $\theta$. Since translates of a coboundary are still coboundaries, we choose $I=[0,\{n \theta\})$ for the sake of simplicity. 
This does not necessarily yield a coboundary, but it is a constant multiple of a coboundary since the equation

$$
\exp \left(\pi i \chi_{I}(x)\right)=\lambda \overline{q(x)} q(x+\theta)
$$

for $\lambda=-e^{\pi i \theta}, q(x)=e^{-\pi i x}, 0<x<1$, implies

$$
\exp \left(\pi i \chi_{[0,\{n \theta\})}(x)\right)=\nu \overline{p(x)} p(x+\theta)
$$

where $\nu=(-1)^{[n \theta]+n} e^{n \pi i \theta}$ and

$$
p(x)=q(x) q(x-\theta) \cdots q(x-(n-1) \theta) .
$$

If $[n \theta]+n$ and $n$ are even, then

$$
\left.\exp \left(\pi i \chi_{[0,\{n \theta\}}\right)(x)\right)=\overline{r(x)} r(x+\theta)
$$

for $r(x)=p(x) e^{n \pi i x}$; hence, it is a coboundary. Otherwise, $\exp \left(\pi i \chi_{[0,\{n \theta\})}\right)$ is not a coboundary. For more details on the construction of coboundaries, see $[1,11,12]$.

Helson [6,7] showed that the spectral type of the unitary operator $U$ in $L^{2}(\mathbb{T})$ defined by $U f(x)=A(x) f(x+\theta),|A(x)|=1$ a.e., is pure, that is, it is purely discrete or purely singular continuous or purely absolutely continuous. In other words, the maximal spectral type of $U$ is pure. For, if we let $L^{2}(\mathbb{T})=$ $H_{\mathrm{ac}} \oplus H_{\mathrm{sc}} \oplus H_{\mathrm{d}}$ be the decomposition into three $U$-invariant subspaces such that $U$ has absolutely continuous spectrum in $H_{\text {ac }}$ and singular continuous spectrum in $H_{\mathrm{sc}}$ and discrete spectrum in $H_{\mathrm{d}}$, then each subspace is invariant under the multiplication by $e^{2 \pi i x}$ and the irrational rotation by $\theta$. Hence each subspace is either the whole space $L^{2}(\mathbb{T})$ or the trivial subspace $\{0\}$. It is also known that, if it is absolutely continuous, then it is Lebesgue.

In [2] it was shown that for sufficiently small rational $0<r$ the unitary operator given by $A(x)=e^{2 \pi i r x}, 0<x<1$, has singular continuous spectrum. In [3] it is shown that, if $A(x)$ is a $C^{2}$-function of modulus 1 with winding number $m \neq 0$, then $U$ has Lebesgue spectrum and that, if $A(x)$ is in $C^{1}$ with $m=0$, then the spectral type of $U$ is singular. Recently, it is shown that, if $A(x)$ is absolutely continuous with winding number $m \neq 0$, then $U$ has Lebesgue spectrum [8]. It is also shown in the same paper that the continuity of $A$ alone does not classify the spectral type of $U$. In [5] it is proved that if $A$ is a step function and if $\theta$ has unbounded partial quotients in its continued fraction expansion, then the spectral type of $U$ is singular. It is not known whether we can have the same result for any arbitrary irrational $\theta$. However, using the same method in [3] we can show that, if $A$ is a step function with its discontinuities only at two points, the spectral type is singular for any irrational $\theta$. In [4] it is proved that, if $A$ is a step function with its discontinuities at rational points, then the spectral type is continuous.

\section{NORMAL NUMBERS}

Consider the measure-preserving transformation $\tau_{2}$ on $\mathbb{T}$ defined by $\tau_{2} x=$ $2 x$. Note that $\tau_{2}$ is strongly mixing. The classical theorem of Borel on normal numbers states that, for almost every $x \in \mathbb{T}$, the number of 1 's in the first $n$ digits of the binary expansion of $x$ is approximately $\frac{1}{2}$, that is,

$$
\lim _{n \rightarrow \infty} \frac{1}{n} \sum_{k=0}^{n-1} \chi_{[1 / 2,1)}\left(\tau^{k} x\right)=\frac{1}{2} \text {. }
$$


This follows from the ergodicity of $\tau_{2}$. As in the previous example we consider Borel's theorem modulo 2. Define $U f(x)=\exp \left(\pi i \chi_{[1 / 2,1)}(x)\right) f\left(\tau_{2} x\right)$. Since $U$ is an isometry, Proposition 2(ii) and the following lemma imply that $\frac{1}{N} \sum_{1}^{N} U^{n} 1$ converges to 0 in $L^{2}(\mathbb{T})$.

Lemma 1. Let $H=\left\{h \in L^{2}(X): U h=h\right\}$. Then $\operatorname{dim} H=0$.

Proof. Suppose $\operatorname{dim} H=1$. Then Proposition 2(ii) implies that

$$
\exp \left(\pi i \chi_{[1 / 2,1)}(x)=q(x) q(2 x)\right.
$$

for some $q, q(x) \in\{1\}$. Replacing $x$ by $1-x$ we get

$$
-\exp \left(\pi i \chi_{[1 / 2,1)}(x)\right)=q(1-x) q(2(1-x)) \text {. }
$$

Multiplying (4) and (5), we get

$$
-1=q(x) q(1-x) q(2 x) q(2(1-x)), \quad(-1) q(x) q(1-x)=q(2 x) q(2(1-x)) .
$$

Hence $\tau_{2}$ has an eigenvalue other than 1 , which contradicts the fact that $\tau_{2}$ is mixing.

Note that $\tau_{2}$ on $\mathbb{T}$ is isomorphic with the one-sided shift on $X=\prod_{1}^{\infty}\{0,1\}$ modulo measure zero sets since $\tau_{2}\left(\sum_{n=1}^{\infty} a_{n} 2^{-n}\right)=\sum_{n=1}^{\infty} a_{n+1} 2^{-n}$. Now we consider different $\tau_{2}$-invariant probability measures other than the Lebesgue measure on $\mathbb{T}$ in the following way: For any $0<p<1$ we define a continuous measure $\mu_{p}$ on $\{0,1\}$ by $\mu_{p}(0)=p, \mu_{p}(1)=1-p$. If $p=1 / 2$, then $\mu_{p}$ is nothing but the Lebesgue measure and any two of such measures with different $p$ 's are mutually singular. We give the product measure on $X$ where on every $\{0,1\}$ some fixed $\mu_{p}$ is given. This is a coin-tossing problem with different odds for head and tail. Now we identify $X$ with $\mathbb{T}$ and consider $U f(x)=\exp \left(\pi i \chi_{[1 / 2,1)}(x) f\left(\tau_{2} x\right)\right.$ in $L^{2}\left(X, \mu_{p}\right)$. Since the one-sided shift is mixing for any $p$, we apply the previous method to prove that the invariant space $H$ is $\{0\}$. Therefore we may conclude that modulo 2 theorem on normal numbers is true for any measure $\mu_{p}$.

Note that for the set $I=\left[\frac{1}{4}, \frac{3}{4}\right)$ the function $q=\exp \left(\pi i \chi_{I}\right)$ is a coboundary since $I=E \Delta \tau_{2}^{-1} E$ for $E=\left[\frac{1}{2}, 1\right)$. But we have $\int q d x=0$; hence, we obtain the uniform distribution modulo 2 in this case even though the invariant subspace is not zero-dimensional.

\section{ACKNOWLEDGMENT}

The author wants to thank Professor Henry Helson for his advice and warm encouragement over many years and to congratulate him on his 65 th birthday.

\section{REFERENCES}

1. G. H. Choe, Ergodicity and irrational rotations, Proc. Roy. Irish Acad Sect. A (to appear).

2. __ Products of operators with singular continuous spectra, Proc. Sympos. Pure Math., vol. 51, part 2, Amer. Math. Soc, Providence, RI, 1990, pp. 65-68.

3. _ Ph.D. Thesis, University of California, Berkeley, 1987.

4. J. P. Conze, Remarques sur les transformations cylindriques et les equations fonctionnelles, Séminaire de Probabilité I, Rennes, France, 1976. 
5. P. Gabriel, M. Lemańczyk, and P. Liardet, Ensemble d'invariants pour les produits croiss de Anzai, Mem. Soc. Math. France 47 (1991).

6. H. Helson, Cocycles on the circle, J. Operator Theory 16 (1986), 189-199.

7. __, The spectral theorem, Lecture Notes in Math., vol. 1227, Springer-Verlag, New York, 1986.

8. A. Iwanik, M. Lemańczyk, and D. Rudolph, Absolutely continuous cocycles over irrational rotations, preprint, 1991.

9. A. Ya. Khinchin, Continued fractions, Univ. of Chicago Press, Chicago, 1964.

10. H. Medina, Hilbert space operators arising from irrational rotations on the circle group, Ph.D Thesis, University of California, Berkeley, 1992.

11. K. D. Merrill, Cohomology of step functions under irrational rotations, Israel J. Math. 52 (1985), 320-340.

12. M. Stewart, Irregularities of uniform distribution, Acta Math. Sci. Hungar. 37 (1981), 185-221.

13. W. A. Veech, Strict ergodicity in zero dimensional dynamical systems and Kronecker-Weyl theorem mod 2, Trans. Amer. Math. Soc. 140 (1969), 1-33.

Department of Mathematics, Korea Advanced Institute of Science and Technology, 373-1 Kusongdong, Yusonggu, TAEjon 305-701, Korea

E-mail address: choeg@math1.kaist.ac.kr 